\title{
Wind farm layout optimization to maximize total power production
}

\author{
I Ulku ${ }^{1}$, C Alabas-Uslu ${ }^{2}$ \\ ${ }^{1}$ Department of Industrial Engineering, Istanbul Kultur University, Istanbul, 34156, Turkey \\ ${ }^{2}$ Department of Industrial Engineering, Marmara University, Istanbul, 34722, Turkey
}

\begin{abstract}
With the rapid increase in energy demand, renewable energy has been considered as an alternative energy resource around the world. A new nonlinear mathematical model is developed in the presented study and a comparison is obtained with a mathematical modelling study from the literature. The results superior according to power generation under a single wind direction. Jensen's wake decay model is used to state multiple wake effect among the turbines. The power production is maximized in terms of the minimization of wake effects. Mathematical formulations are represented and compared with the model from the literature. The generated layouts are presented and expected power among the Introduction turbines are significantly improved.
\end{abstract}

\section{Introduction}

Renewable energy is considered as an alternative energy resource because of its high potential to provide the required energy capacity. Continuity and sustainability opportunities of renewable energy is noticed and used as an attractive source in the developed countries. Wind power extracts the kinetic energy from the wind and converts it into electricity with the help of turbines. Turbulence or wake is generated behind the tower, while it is working. Thus, power production and the wind speed deficits of a wind turbine are reduced by another turbine's wake in the wind farm. Electricity generation with the wind farm development takes a meaningful impact with the correct estimation of the wind power. Therefore, not only the location selection, but also the placement of turbine is crucial for investment on a wind farm, in terms of maximizing power production with minimizing wake effect among the turbines.

A new nonlinear mathematical programming model is proposed where totally unimodularity property is used in the model for wind farm layout problems. The aim is to maximize total generated power while minimizing the wake effect among the located turbines. An interaction matrix is generated priori as presented in Turner et al. [1] and several number of wind turbines are tested. Wind farms are expensive investments and improvements in the investment cost will be quite valuable. The objective function where total power produced is nonlinear and binary variables of wind turbine locations make the model to be complex. In the presented model, the totally unimodularity property is introduced. Thus, the optimum turbine locations are binary without using integer programming model and generates higher total power production for a predetermined turbine numbers.
In Section 2, literature review is introduced, and nonlinear mathematical programming model is represented in section 3. The performance of the model, conclusions and future study details are is given in section 4 and 5, respectively.

\section{Literature Review}

Jensen in 1983 [2] has developed one of the most popular wake models. Jensen model is based on the distance behind the rotor and it is a linear expanding wake with the velocity deficit which is widely used in the literature. Figure 1 illustrates a single wind turbine wake effect. The wind blows with a speed $u_{0}$ from the left and passes through the wind turbine 1 with the rotor radius $r$. Wind turbine 2 is affected from the wake created by the turbine 1. Genetic algorithm is used mostly in the literature as heuristics to solve wind farm layout problems [3-5]. First model based on genetic algorithm is introduced by Mosetti et al. [3] to find optimal placement of the wind turbines. Jensen's wake decay model is used to analyse the wake effect according to various wind speeds and directions. A test case is used by dividing a square into 100 square cells where possible turbine locations can be arranged. Grady et al. [4] developed a genetic algorithm to find optimal placement of turbines, where the number of installed turbines are limited. A Monte Carlo simulation method determines optimal turbine placement in the study of Marmidis et al. [5]. Turner et al. [1] presented two mathematical models to determine the efficiency between them. First model is a quadraticinteger model and second model is a linear-integer programming model. With these models an interaction matrix is prepared priori, to determine wake effects on each possible wind turbine location. 
A set of possible turbine locations are $(i, j)$ pairs for all $i, j \in I$ where $I=\{1, \ldots, N\}$ is the index set. Distance between turbines at position $(i, j)$ and $(k, l)$ for $\mathrm{i}, \mathrm{j}, \mathrm{k}, \mathrm{l} \in$ I is represented as $D_{i j k l}$. The wake radius $r_{1}$ and the

$$
\begin{gathered}
r_{1}=\alpha \cdot D_{i j k l}+r \\
\alpha=\frac{0.5}{\ln \frac{Z}{z_{0}}}
\end{gathered}
$$

wake enlargement, $\alpha$ are calculated in equation (1) and (2), respectively. Coefficients $z$ represents hub height and $z_{0}$ indicates surface roughness [2].

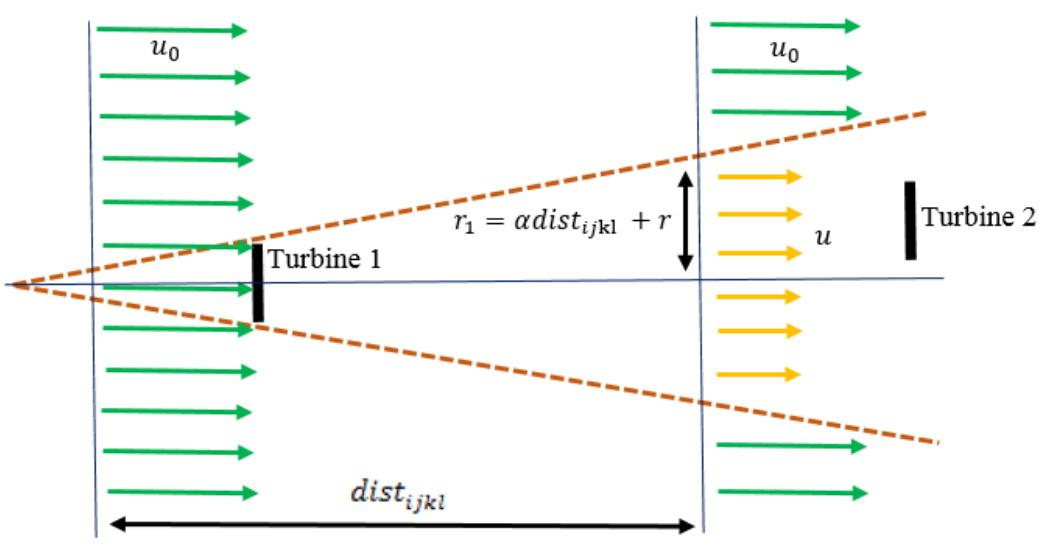

Figure 1. Wake effect representation

Wind speed, $u_{i j}$ at position $(i, j)$ is determined by equation (3) according to Jensen's wake model as $V d_{i j k l}$ is the velocity deficit in the wind speed due to the turbine located at $(k, l)$ and $u_{0}$ is the ambient wind speed.

$$
\begin{gathered}
u_{i j}=u_{0}\left(1-V d_{i j k l}\right) \\
V d_{i j k l}=\frac{2 \cdot a}{\left(1+\frac{\alpha \cdot D_{i j k l}}{r}\right)^{2}} \\
a=0.5\left(1-\sqrt{1-C_{T}}\right)
\end{gathered}
$$

Equation (4) is used to calculate $V d_{i j k l}$ by using rotor radius $r$, distance $D_{i j k l}$ and axial induction factor $a$ which is dependence of thrust coefficient, $C_{T}$ as given by equation (5).
Multiple wake effects occur when a turbine is located at position $(i, j)$ and affected by more than one turbine's wake. Thus, sum of the squares of individual velocity deficits generated by each wake is equal to $V d e f_{i j}$ as shown in equation (6) [2]. Equation (7) represents the remaining velocity $u_{i j}$ by a turbine at $(i, j)$.

$$
\begin{array}{r}
\mathrm{Vdef}_{i j}=\sqrt{\sum_{i=1}^{N} \sum_{j=1}^{N} \sum_{k=1}^{N} \sum_{l=1}^{N} \mathrm{Vd}_{i j k l}^{2}} \\
u_{i j}=u_{0} \cdot\left[1-\mathrm{Vdef}_{i j}\right]
\end{array}
$$

\section{Mathematical Programming Framework}

In a wind farm layout wake effect has a key factor on the total power production since, the wind speed is decreased by individual wind turbines as explained in Section 2. A new nonlinear mathematical model is proposed under a single wind direction for the wind farm layout problem. An available wind farm is represented with a grid of size $N^{2}$ in which each cell $(i, j)$ corresponds a possible turbine location and the number of turbines, $\mathrm{TN}$, to be placed in the wind farm is known in advance. Interaction matrix of size $\left(N^{2}\right) x\left(N^{2}\right)$ is created with all possible multiple wake effects. The turbines are assumed that can turn to face at any given wind direction. Each possible turbine location has $J-1$ interaction between itself and other possible 
turbine locations. A decision variable for wind farm layout problem is as follows:

$$
x_{i j}= \begin{cases}1, & \text { if there is a turbine at position }(\mathrm{i}, \mathrm{j}) \text { for } i, j=1, \ldots, N \\ 0, & \text { otherwise }\end{cases}
$$

Since wind turbine efficiency, $40 \%$, and hub radius, $20 \mathrm{~m}$, are used in the related literature [7] the coefficient of 0.3 in equations (8) is used. To maximize total power production where the power produced by individual wind turbines $(i, j)$ is indicated with equation $(10)$ for wind farm layout problem. TP, total sum of the power is shown in equation (9) that is produced by all turbines located at positions $(i, j)$. The nonlinear integer mathematical model of wind farm layout problem, is given by equations 10-12. Total number of turbines are known and satisfied with equation (11) since binary conditions of variables are represented in equation (12).

$$
\begin{gathered}
P_{i j}=(0.3) \cdot u_{i j}^{3} \\
T P=\left\{\sum_{i=1}^{N} \sum_{j=1}^{N} P_{i j} \quad \text { for } x_{i j}=1, \forall i, j\right\} \\
\operatorname{Max} \sum_{i=1}^{N} \sum_{j=1}^{N} P_{i j} \cdot x_{i j} \\
\sum_{i=1}^{N} \sum_{j=1}^{N} x_{i j}=T N \\
x_{i j} \in(0,1) \quad \forall i, j
\end{gathered}
$$

There are some obstacles during the solution procedure of the wind farm layout. Firstly, due to the nonlinearity, the equation (10) is a main difficulty to solve the layout problem. Turner et al. [1] proposed a linearized integer programming model by minimization of total velocity deficits to handle this difficulty. Secondly,

$$
0 \leq x_{i j} \leq 1 \text { for } \forall i, j
$$

Although the constraints does not take 0-1 binary values, an optimum layout to the problem is also satisfied as the binary requirements. With this relaxed form of the problem, search space is decreased and proposed nonlinear integer model with equations (10), (11) and (13) is solved simpler than the model through equations (10)(12). The quadratic integer model of Turner et al. [1] has to locate a turbine or not for each possible turbine locations in a grid with size 100 consists of a huge search space. Therefore, the binary constraints in equation (12) are replaced with equation (13) with the help of totally unimodular matrix property [6].

the following objective function where sum of the multiple wake effects are minimized as in equation (14). The quadratic integer model of Turner et al. [1] is represented with equations (14), (11), and (12) and compared with the proposed nonlinear integer model in this study.

$$
\operatorname{Min} \sum_{i=1}^{N} \sum_{j=1}^{N} \sum_{k=1}^{N} \sum_{l=1}^{N} x_{i j} * x_{k l} * V d e f_{i j}
$$

\section{Computational Results}

A set of test problems are generated to represent the performance of the proposed nonlinear model for single wind direction from north to south. The parameters in table 1 are used in this study. An interaction matrix is prepared priori in order to determine velocity deficits of each possible turbine locations.

Table 1. Input parameters for the cases.

\begin{tabular}{cc}
\hline Grid size & 100 square cells \\
Cell size & $10 r_{0}(200 \mathrm{~m} \times 200 \mathrm{~m})$ \\
Wind speed $\left(u_{0}\right)$ & $12 \mathrm{~m} / \mathrm{s}$ \\
Rotor radius $\left(r_{0}\right)$ & $20 \mathrm{~m}$ \\
Hub height $\left(z_{0}\right)$ & $60 \mathrm{~m}$ \\
\hline
\end{tabular}




\begin{tabular}{ccc}
\hline Thrust coefficient $\left(C_{T}\right)$ & 0.88 & \\
Entrainment constant $(\alpha)$ & 0.1 & \\
Power $\left(P_{i j}\right)$ & $P_{i j}=(0.3) * u_{i j}^{3}$ & Ref. [3] \\
\hline
\end{tabular}

In the following subsection, the obtained expected power results from the proposed nonlinear model are compared with the quadratic integer model by Turner et al. [1].

\subsection{Comparison of Non-Linear Model with Quadratic Integer Model}

Both non-linear model and quadratic integer model are performed in LINGO [8] on a $1.70 \mathrm{GHz}$ Intel Core i5 notebook with 6 GB of RAM with an upper limit of 2 hours on the run time. Thus, total power results of nonlinear model are the lower bounds on the exact optimum power for the test cases of wind farm layout problem. Generated total power results by non-linear model and quadratic integer model are introduced in Table 2 for several number of turbines considering single wind direction from north to south. Although for 10 and 20 turbines both models obtain the same total power, nonlinear model has better power output than quadratic integer model for 30 and more turbine layout. Apparently, as the number of turbines increases the performance of non-linear model proposed in this study also increases comparing the quadratic integer model. Since the objective function of the non-linear model is to maximize total power generated, this model is more successful than the quadratic model which minimizes the total velocity deficits. Although, minimization of total velocity deficits is necessary to generate more power, this study shows that if the objective function is selected as maximization of total power instead of minimizing velocity deficits, the resulting wind farm layouts will be more efficient.

Table 2. Single Wind Direction from north to south

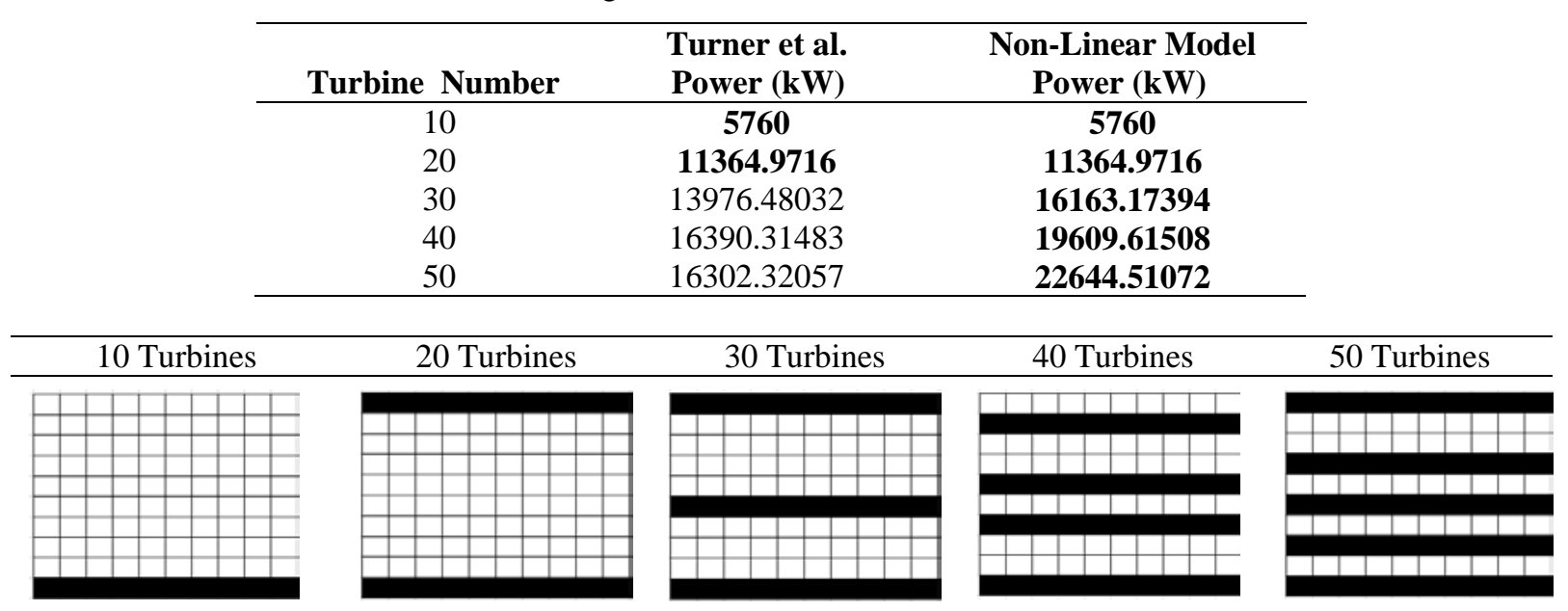

Figure 2. Non-Linear Model Layouts for a Single Wind Direction

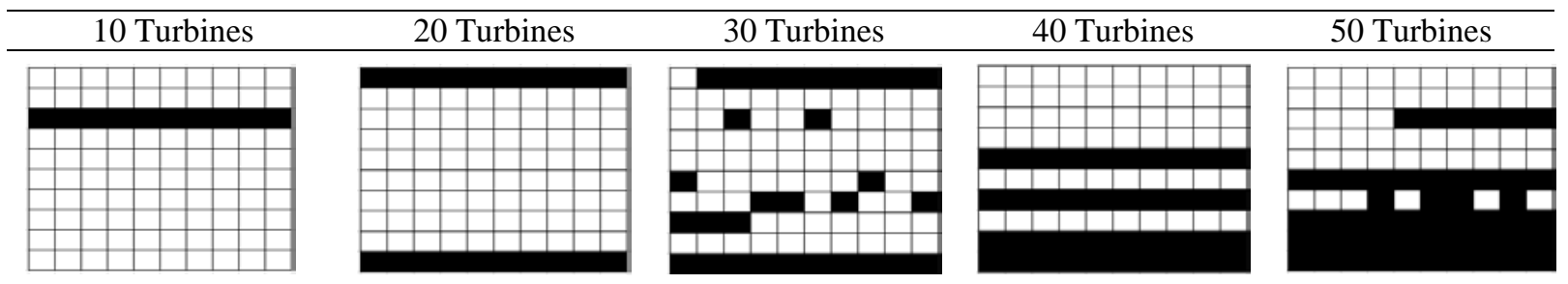

Figure 3. Quadratic Integer Model Layouts for a Single Wind Direction

Figure 2 and Figure 3 gives the optimal wind farm layouts of non-linear model and quadratic integer model for the turbine numbers from 10 to 50 turbines under single wind direction.

\section{Conclusion}

A new nonlinear mathematical model is presented to solve layout of wind farm problem to maximize total power production in terms of multiple wake effects. Jensen's multiple wake decay model is used for the presented mathematical model. Due to nonlinearity of the power and wake effects calculations the researchers preferred to use heuristic methods in the literature. Layout of a wind farm is a permanent investment, thus, any improvement in the layout of a wind farm increase the generated total power. The proposed nonlinear mathematical programming model has totally unimodularity property where binary variables of wind 
turbine locations is relaxed and model is solved simpler. It is obvious that solving the proposed nonlinear model within a limited run time generates better lower bounds on the exact optimum power in comparison with the quadratic integer model [1]. Also, an interaction matrix is used priori as presented in Turner et al. [1] and various number of wind turbines are performed. The proposed study express that the wind farm layout problems can have optimum layouts. We are planning to extend our study by including multiple wind direction. Thus, various situations can be handled for the wind farm layout problems.

\section{References}

1 Turner SDO, Romero DA, Zhang PY, Amon CH, Chan TCY. A new mathematical programming approach to optimize wind farm layouts, 2014, Renewable Energy. 674-680.

2 Jensen NO. A note on wind generator interaction RISO National Laboratory; 1983 November; Roskilde, DK-4000, Denmark.
3 Mosetti G, Poloni C, Daviacco B. Optimization of wind turbine positioning in large windfarms by means of a genetic algorithm. 1994, J Wind Eng Ind Aerod. 51:105-116.

4 Grady S, Hussaini M, Abdullah M. Placement of wind turbines using genetic algorithms. 2005, Renewable Energy. 30: 259 - 270.

5 Marmidis G, Lazarou S, Pyrgioti E. Optimal placement of wind turbines in a wind park using monte carlo simulation, 2008, Renewable Energy. 33: 1455 - 1460.

6 Wolsey LA. Integer Programming, Wiley, 1998.

7 Mittal A. Optimization of the Layout of Large Wind Farms using a Genetic Algorithm. Department of Mechanical and Aerospace Engineering, 2010, Case Western Reserve University, Cleveland.

8 Extended LINGO/Win 64. Release 17.0.65. Lindo System Inc. Chicago, September 2017. 